\title{
Translational Coupling in Escherichia coli of a Heterologous Bacillus subtilis-Escherichia coli Gene Fusion
}

\author{
TAHA I. ZAGHLOUL AND ROY H. DOI* \\ Department of Biochemistry and Biophysics, University of California at Davis, Davis, California 95616
}

Received 2 June 1986/Accepted 13 August 1986

The efficient expression in Escherichia coli of the Tn9-derived chloramphenicol acetyltransferase (EC 2.3.1.28) gene fused distal to the promoter and $\mathrm{N}$ terminus of the Bacillus subtilis aprA gene was dependent on the initiation of translation from the ribosome-binding site in the aprA gene.

Translational coupling (14) in Escherichia coli $(1,3,15,16)$ and in Bacillus subtilis $(18,23)$ has been observed. In the usual case of translational coupling, the expression of a distal gene in an operon is dependent on the translation of a proximal gene which has a translation stop codon close to or even overlapping the initiation codon of the distal gene. The exact mechanism of translational coupling is not clearly understood, although several models have been proposed (14).

We found that a inefficiently used gram-negative ribosome-binding site (RBS) could be used much more efficiently in $B$. subtilis if translation of a $B$. subtilis-E. coli fusion mRNA was initiated at the $B$. subtilis RBS and if the translation of the proximal $B$. subtilis gene terminated within the gram-negative RBS of the distal $E$. coli gene (23).

Using the same $B$. subtilis- $E$. coli gene fusions, we investigated whether translation coupling occurred in $E$. coli and whether the mechanism was similar to that found in $B$. subtilis. Since the distal Tn9-derived chloramphenicol acetyltransferase (cat [ptsG]) gene has a gram-negative RBS (6), the RBS should be used efficiently in $E$. coli and not be dependent on translation of the proximal $B$. subtilis gene unless some type of transcription-translation coupling also occurs.

The $B$. subtilis- $E$. coli gene fusion was constructed between the $\mathbf{N}$ terminus of the $B$. subtilis subtilisin gene ( $S$ fragment) $(7,22)$ and the promoterless $T n$ 9-derived cat gene to form the shuttle plasmid pGR71S $(5,7,21,23)$. The $S$ fragment contains the promoters, RBS, signal peptide sequence, propeptide sequence, and the $\mathrm{N}$-terminal 49 amino acids of the mature subtilisin protein (22). When this shuttle plasmid was transformed into $E$. coli $\operatorname{HB} 101(\lambda)(10)$, the specific activity of chloramphenicol acetyltransferase (CAT) $(9,17)$ remained constant during all phases of growth (data not shown).

Translation initiation in pGR71S can occur either from the gram-positive RBS of subtilisin and continue into the gramnegative RBS of the cat gene in frame with two translation stop codons or from the gram-negative RBS of the cat gene (23). The product made from this construction is a nativesized CAT product, and the activity is low when compared with that found in B. subtilis (Fig. 1) (23).

To see whether translational coupling was occurring in this gene fusion, we deleted a 178-base-pair (bp) HpaI fragment from the $S$ fragment (pGR71S $\Delta 3$ ), which simultaneously created a translational termination codon $230 \mathrm{bp}$ upstream of the gram-negative RBS of the cat gene (23). The

\footnotetext{
* Corresponding author.
}

presence of this deletion resulted in the lowering of cat gene expression by about $50 \%$ (Fig. 1, line 2). To test whether this lower activity was the result of disruption of translational coupling, we inserted a 10-base BamHI linker into the $\mathrm{HpaI}$ deletion site (pGR71S $\Delta 4$ ), which simultaneously restored the open reading frame, removed the translation stop codon, and allowed the translation to continue to the translation termination codon in the RBS of the cat gene (Fig. 1, line 3) (23). When CAT activity was tested, a fivefold higher activity was observed for pGR71S $\Delta 4$ than for pGR71S $\Delta 3$ (Fig. 1; compare lines 2 and 3 ).

As a control, a fragment containing only the promoter of the subtilisin gene $(a p r A)$ was inserted in front of the cat gene (pGR71Papr) (23). In this construct, translation could be initiated only from the cat gene RBS. The CAT activity from this construction (Fig. 1, line 4) was higher than that found with pGR71S and pGR71S $\Delta 3$ but lower than that with pGR71 $\Delta 4$. In all of the constructs, only native-sized CAT was produced as determined by sodium dodecyl sulfatepolyacrylamide gel electrophoresis (8) and Western blot analysis (data not shown) (23).

To show that the differences in cat gene expression were not due to variations in the level of CAT mRNA, we measured the amounts of mRNA being transcribed from the fusion genes with an anti-CAT mRNA probe. The probe was constructed by inserting the HindIII-Sall cat gene (5) into the SalI-HindIII site of plasmid pSP65 (13), followed by in vitro transcription of this template plasmid with SP6 RNA polymerase (13). As an internal control for the amount of mRNA being made from the constructs, $a \mathrm{Km}^{\mathrm{r}}$ mRNA probe ( $\mathrm{Km}^{\mathrm{r}}$ mRNA is mRNA from the $\mathrm{Km}^{\mathrm{r}}$ gene) was used to eliminate differences in plasmid copy number, in mRNA isolation, and in relative decay of different $m R N A$ sizes. The probe was constructed by cloning a 400-bp HincII-BglII fragment (this part of the $\mathrm{Km}^{\mathrm{r}}$ gene is carried by plasmid pGR71 [5]) in the HincII-BamHI sites of plasmid pSP65. These probes were used to measure the amounts of CAT mRNA and $\mathrm{Km}^{\mathrm{r}}$ mRNA $(2,4,11,12,19)$ produced by the different pGR71 constructs (23) (Table 1).

The results in the last column of Table 1 , which are based on normalized CAT mRNA concentrations, have almost the same relative values as the specific activity data for CAT. These results indicate that transcriptional differences were not the reason for the variations observed in cat gene expression. The most significant difference in CAT expression was seen between pGR71S $\Delta 3$ and pGR71S $\Delta 4$. The translation in pGR71 $\Delta 3$ is terminated 230 bases upstream of the cat RBS, whereas in pGR71S $\Delta 4$, the translation is continued to the RBS of the cat gene. The only difference 


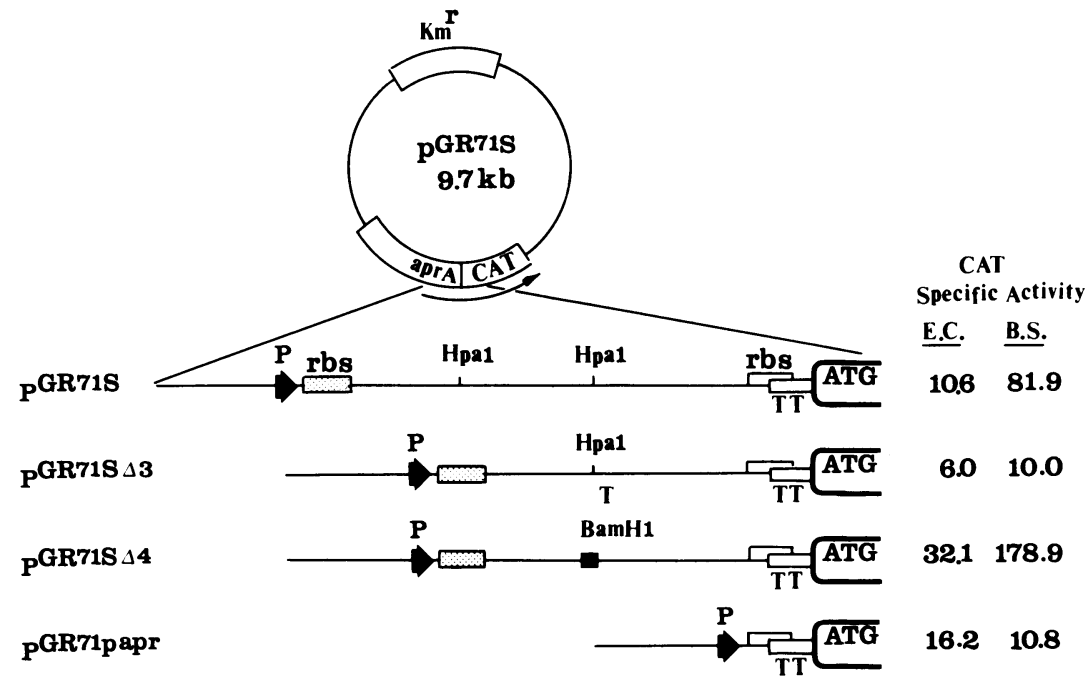

FIG. 1. A schematic representation of the fusion between the $\mathrm{N}$ terminus of the aprA gene and the cat gene present in plasmid pGR71S. The construction of the plasmids has been described by Zaghloul et al. (23). $\square$, RBS of B. subtilis; $\square$, RBS of E. coli; TT, translation termination sites in the RBS of the cat gene; T, translation termination site resulting from the 178-bp HpaI deletion in pGR71S $\Delta 3$; $B a m H I$ linker added into the $H p a I$ site, resulting in the elimination of the translational termination site in pGR71S $\Delta 4 ;, a p r A$ promoter (which is found alone [no $B$. subtilis RBS] in front of the cat gene in pGR71Papr). The data for $B$. subtilis CAT-specific activity are from Zaghloul et al. (23). E.C., E. coli; B.S., B. subtilis.

between these two constructs is the 10-base BamHI insert in pGR71S $\Delta 4$, which eliminates the termination codon and restores the open reading frame up to the cat RBS. These results strongly suggest that translational coupling, not coupled premature transcription termination, is the major reason for the difference observed between pGR71S $\Delta 3$ and pGR71S $\Delta 4$.

The three- to fourfold higher activity of pGR71S $\Delta 4$ over pGR71S and the mRNA data (Table 1) suggest that the shorter translational distance between the aprA and cat RBS allows more efficient expression of the cat gene. The results do not support the presence of a putative transcriptional pause site in the $168 \mathrm{bp}$ which were deleted.

An enhancing role for multiple RBSs is suggested by the greater CAT activity seen with pGR71S 44 than with pGR71Papr, since the pGR71S 4 mRNA has both the aprA and cat RBSs, whereas pGR71Papr mRNA has only the cat RBSs.

The CAT activities of the constructs in $E$. coli are lower because $B$. subtilis aprA promoters, which are weakly expressed in $E$. coli (20), control the expression of the heterologous gene fusion.

TABLE 1. Level of CAT expression in $E$. coli cells carrying recombinant plasmids normalized to mRNA content

\begin{tabular}{lccccc}
\hline & \multicolumn{5}{c}{ Relative level of CAT expression } \\
\cline { 2 - 6 } Plasmid & $\begin{array}{c}\text { Km }^{\mathrm{r}} \\
\text { mRNA }^{a}\end{array}$ & $\begin{array}{c}\text { CAT } \\
\text { mRNA }\end{array}$ & $\begin{array}{c}\text { CAT } \\
\text { mRNA/Km } \\
\text { mRNA }\end{array}$ & $\begin{array}{c}\text { Sp act of } \\
\text { CAT }\end{array}$ & $\begin{array}{c}\text { Sp act/ } \\
\text { normalized } \\
\text { CAT } \\
\text { mRNA }\end{array}$ \\
\hline pGR71S & 2.10 & 1.36 & 0.52 & 10.6 & 20.4 \\
pGR71S $\Delta 3$ & 1.00 & 1.00 & 1.00 & 6.0 & 6.0 \\
pGR71S $\Delta 4$ & 2.70 & 1.00 & 0.40 & 32.1 & 80.2 \\
pGR71Papr & 1.04 & 1.13 & 1.10 & 16.1 & 14.6 \\
\hline
\end{tabular}

${ }^{a}$ Relative level of $\mathrm{Km}^{\mathrm{r}} \mathrm{mRNA}$ as determined by hybridization to anti-Km ${ }^{\mathrm{r}}$ mRNA probe. Values are relative to that of pGR71S $\Delta 3$.

$b$ Values derived as described in footnote $a$, except hybridization was to anti-CAT mRNA probe.
Our results indicated that although translation from the gram-negative RBS did occur and a native-sized product was synthesized as expected, the efficiency of translation was greater if translation was initiated from the RBS of the upstream $B$. subtilis gene and if the ribosomes terminated translation in the gram-negative RBS of the cat gene. Thus, translational coupling is necessary for efficient expression of the cat gene in the heterologous fusion even in the gramnegative host.

This research was supported in part by National Institute of General Medical Sciences grant GM 19673-14 and National Science Foundation grant PCM82-18304.

We thank Fujio Kawamura and Sui-Lam Wong for their advice and assistance.

\section{LITERATURE CITED}

1. Aksoy, S., C. L. Squires, and C. Squires. 1984. Translational coupling of the $\operatorname{trpB}$ and $\operatorname{trpA}$ genes in the Escherichia coli tryptophan operon. J. Bacteriol. 157:363-367.

2. Berent, S. L., M. Mahmoudi, R. M. Torezynski, P. W. Bragg, and A. P. Bollon. 1985. Comparison of oligonucleotide and long DNA fragments as probes in DNA and RNA dot, Southern, Northern, colony, and plaque hybridizations. BioTechniques 3:208-220.

3. Das, A., and C. Yanofsky. 1984. A ribosome binding site sequence is necessary for efficient expression of the distal gene of a translationally coupled gene pair. Nucleic Acids Res. 12:4757-4768.

4. Gilman, M. Z., and M. J. Chamberlin. 1983. Developmental and genetic regulation of the Bacillus subtilis genes transcribed by sigma-28 RNA polymerase. Cell 35:285-293.

5. Goldfarb, D. S., R. H. Doi, and R. L. Rodriguez. 1981. Expression of Tn9-derived chloramphenicol resistance in Bacillus subtilis. Nature (London) 293:309-311.

6. Goldfarb, D. S., R. L. Rodriguez, and R. H. Doi. 1982. Translational block to expression of the Escherichia coli $\mathrm{Tn}$ 9-derived chloramphenicol-resistance gene in Bacillus subtilis. Proc. Natl. Acad. Sci. USA 79:5886-5890.

7. Goldfarb, D. S., S. L. Wong, T. Kudo, and R. H. Doi. 1983. A 
temporally regulated promoter from Bacillus subtilis is transcribed only by an RNA polymerase with a 37,000 dalton sigma factor. Mol. Gen. Genet. 191:319-325.

8. Laemmli, U. K. 1970. Cleavage of structural proteins during the assembly of the head of bacteriophage T4. Nature (London) 227:680-685.

9. Lowry, O. H., N. J. Rosebrough, A. L. Farr, and R. J. Randall. 1951. Protein measurement with the Folin phenol reagent. J. Biol. Chem. 193:265-275.

10. Maniatis, T., E. F. Fritsch, and J. Sambrook. 1982. Molecular cloning: a laboratory manual. Cold Spring Harbor Laboratory, Cold Spring Harbor, N.Y.

11. Matthews, H. 1984. Computer analysis of gel scans. In J. M. Walker (ed), Methods in molecular biology, vol. 1. Proteins. The Humana Press, Inc., Clifton, N.J.

12. Meinkoth, J., and G. Wahl. 1984. Hybridization of nucleic acids immobilized on solid supports. Anal. Biochem. 138:267-284.

13. Melton, D. A., P. A. Krieg, M. R. Rebagliati, T. Maniatis, K. Zinn, and M. R. Green. 1984. Efficient in vitro synthesis of biologically active RNA and RNA hybridization probes from plasmids containing a bacteriophage SP6 promoter. Nucleic Acids Res. 12:7035-7056.

14. Oppenheim, D., and C. Yanofsky. 1980. Translational coupling during expression of the tryptophan operon of Escherichia coli. Genetics 95:785-795.

15. Riggs, D., and S. Artz. 1984. The hisD-hisC gene border of the Salmonella typhimurium histidine operon. Mol. Gen. Genet.
196:526-529.

16. Schumperli, D., K. McKenney, D. A. Sobieski, and M. Rosenberg. 1982. Translational coupling at an intercistronic boundary of the Escherichia coli galactose operon. Cell 30:865-871.

17. Shaw, W. 1975. Chloramphenicol acetyltransferase from chloramphenicol-resistant bacteria. Methods Enzymol. 43:737-755.

18. Sprengel, R., B. Reiss, and H. Schaller. 1985. Translationally coupled initiation of protein synthesis in Bacillus subtilis. Nucleic Acids Res. 13:893-909.

19. White, B. A., and F. C. Bancroft. 1982. Cytoplasmic dot hybridization, simple analysis of relative mRNA levels in multiple small cell or tissue samples. J. Biol. Chem. 257:8569-8572.

20. Wong, S.-L., and R. H. Doi. 1984. Utilization of a Bacillus subtilis $\sigma^{37}$ a promoter by Escherichia coli RNA polymerase in vivo. J. Biol. Chem. 259:9762-9767.

21. Wong, S.-L., D. S. Goldfarb, C. W. Price, and R. H. Doi. 1984. Structure, regulation and genetic locus of a temporally expressed promoter of Bacillus subtilis, p. 209-221. In A. T. Ganesan and J. A. Hoch, (ed.), Genetics and biotechnology of bacilli. Academic Press, Inc., New York.

22. Wong, S.-L., C. P. Price, D. S. Goldfarb, and R. H. Doi. 1984. The subtilisin E gene of Bacillus subtilis is transcribed from a $\sigma^{37}$ promoter in vivo. Proc. Natl. Acad. Sci. USA 81:1184-1188.

23. Zaghloul, T. I., F. Kawamura, and R. H. Dol. 1985. Translational coupling in Bacillus subtilis of a heterologous Bacillus subtilis-Escherichia coli gene fusion. J. Bacteriol. 164:550-555. 